\title{
CO Spectral Line Energy Distributions in Galactic Sources: Empirical Interpretation of Extragalactic Observations*
}

\author{
Nick Indriolo $^{1,7}$, E. A. Bergin ${ }^{1}$, J. R. Goicoechea ${ }^{2}$, J. Cernicharo' ${ }^{2}$, M. Gerin ${ }^{3}$, A. Gusdorf ${ }^{3}$, D. C. Lis ${ }^{4,5}$, and P. Schilke ${ }^{6}$ \\ ${ }^{1}$ Department of Astronomy, University of Michigan, 1085 S. University Avenue, Ann Arbor, MI 48109, USA; nindriolo@stsci.edu \\ ${ }^{2}$ Grupo de Astrofísica Molecular, Instituto de Ciencia de Materiales de Madrid (CSIC) E-28049 Madrid, Spain \\ ${ }^{3}$ LERMA, Observatoire de Paris, PSL Research University, CNRS, Sorbonne Universités, UPMC Univ. Paris 06, École normale supérieure, F-75005, Paris, France \\ ${ }^{4}$ LERMA, Observatoire de Paris, PSL Research University, CNRS, Sorbonne Universités, UPMC Univ. Paris 06, F-75014, Paris, France \\ ${ }^{5}$ California Institute of Technology, Cahill Center for Astronomy and Astrophysics 301-17, Pasadena, CA 91125, USA \\ ${ }^{6}$ I. Physikalisches Institut der Universität zu Köln, Zülpicher Str. 77, D-50937 Köln, Germany \\ Received 2016 November 15; revised 2016 December 30; accepted 2017 January 12; published 2017 February 13
}

\begin{abstract}
The relative populations in rotational transitions of $\mathrm{CO}$ can be useful for inferring gas conditions and excitation mechanisms at work in the interstellar medium. We present $\mathrm{CO}$ emission lines from rotational transitions observed with Herschel/HIFI in the star-forming cores Orion S, Orion KL, Sgr B2(M), and W49N. Integrated line fluxes from these observations are combined with those from Herschel/PACS observations of the same sources to construct CO spectral line energy distributions (SLEDs) from $5 \leqslant J_{u} \leqslant 48$. These CO SLEDs are compared to those reported in other galaxies, with the intention of empirically determining which mechanisms dominate excitation in such systems. We find that CO SLEDs in Galactic star-forming cores cannot be used to reproduce those observed in other galaxies, although the discrepancies arise primarily as a result of beam filling factors. The much larger regions sampled by the Herschel beams at distances of several megaparsecs contain significant amounts of cooler gas, which dominate the extragalactic CO SLEDs, in contrast to observations of Galactic starforming regions, which are focused specifically on cores containing primarily hot molecular gas.
\end{abstract}

Key words: galaxies: ISM - ISM: molecules

\section{Introduction}

The Herschel Space Observatory (Pilbratt et al. 2010) enabled the first surveys of rotational transitions of $\mathrm{CO}$ with $4 \leqslant J_{u} \leqslant 50$ in emission throughout a wide sample of galaxies. These $\mathrm{CO}$ emission lines can be used to place constraints on the physical conditions (e.g., density, temperature, radiation field) within the emitting gas as the relative populations in the various rotational states are controlled by collisional and radiative (de)excitation. The shape of the CO Spectral Line Energy Distribution (SLED) - flux in each emission line as a function of upper state energy-provides information about the gas conditions, and potentially the agent (e.g., shocks, X-rays, UV photons, cosmic rays) primarily responsible for heating the gas. Multiple observing programs targeted $\mathrm{CO}$ emission lines in the central regions of different types of galaxies-e.g., UltraLuminous InfraRed Galaxies, Seyfert galaxies, starburst galaxies-for the purpose of determining which of the aforementioned mechanisms dominate the gas heating in each case. However, in most galaxies the observed CO SLEDs can be fit with a variety of models, such that it is difficult to conclude whether shocks, photon dominated regions (PDRs), or X-ray dominated regions (XDRs), are driving the CO excitation (e.g., Hailey-Dunsheath et al. 2012; Kamenetzky et al. 2014; Mashian et al. 2015; Rosenberg et al. 2015). While kinematic information would provide a clue to this puzzle, both the Spectral and Photometric Imaging Receiver (SPIRE; Griffin et al. 2010) and Photoconductor Array Camera and Spectrometer (PACS; Poglitsch et al. 2010) instruments used for these

\footnotetext{
* Herschel is an ESA space observatory with science instruments provided by European-led Principal Investigator consortia and with important participation from NASA.

7 Current address: Space Telescope Science Institute, Baltimore, MD 21218, USA.
}

extragalactic observations are incapable of spectrally resolving the $\mathrm{CO}$ emission lines.

Herschel observations of $\mathrm{CO}$ emission lines have also been reported for a variety of regions within our Galaxy, including the well-studied objects Sgr B2 (Etxaluze et al. 2013), Sgr A (Goicoechea et al. 2013), and Orion KL (Goicoechea et al. 2015). In some Galactic sources, however, in addition to the low spectral resolution PACS data we also have Herschel Heterodyne Instrument for the Far Infrared (HIFI; de Graauw et al. 2010) observations of $J_{u} \leqslant 16$ transitions of CO that are spectrally resolved. By studying the velocity profiles of these $\mathrm{CO}$ emission lines in Galactic sources we can better constrain the excitation mechanisms involved in producing different $\mathrm{CO}$ SLED shapes.

Galactic regions that we consider herein include the Orion Bar, Orion South, Orion KL, Sgr B2(M), and W49N. The Orion Bar is a prototypical strongly illuminated PDR, and is located in the Orion star-forming region at a distance of $414 \mathrm{pc}$ (Menten et al. 2007). It has the distinction of being nearly "edge-on," such that the atomic and molecular emission from different stratified layers can be studied (e.g., van der Wiel et al. 2009; Nagy et al. 2013). Orion S is an embedded starforming region that contains multiple outflows (Schmid-Burgk et al. 1990; Ziurys et al. 1990; Zapata et al. 2005, 2006), shocked gas (Henney et al. 2007; Rivilla et al. 2013), and a PDR illuminated by the Trapezium stars (Peng et al. 2012). In Section 4.2 we show that the $\mathrm{CO}$ emission profiles in Orion $\mathrm{S}$ can be decomposed into these three components. Orion KL is a luminous, high-mass star-forming region comprised of multiple spatial and kinematic components. It harbors warm gas clumps, multiple suspected protostars, quiescent gas, and shocked gas resulting from explosive outflows (Blake et al. 1987; Zapata et al. 2009, 2011; Nissen et al. 2012; Crockett et al. 2014). 
Table 1

Observation Identifiers (ObsIDs) for Spectra Containing CO Emission

\begin{tabular}{|c|c|c|c|c|}
\hline & Orion $\mathrm{S}$ & Orion KL & Sgr B2(M) & $\mathrm{W} 49 \mathrm{~N}$ \\
\hline & \multicolumn{4}{|c|}{ HIFI } \\
\hline $\mathrm{CO} J=5-4$ & 1342204001 & 1342191592 & 1342191565 & \\
\hline $\mathrm{CO} J=6-5$ & 04708 & 194540 & 192546 & $1342194554,5,6$ \\
\hline $\mathrm{CO} J=7-6$ & 05332 & 205334 & 204723 & $1342230253,4,5$ \\
\hline $\mathrm{CO} J=8-7$ & 05336 & 192329 & 206455 & $1342244816,7,8$ \\
\hline $\operatorname{CO} J=9-8$ & 03150 & 191601 & 218200 & \\
\hline $\operatorname{CO} J=10-9$ & 05871 & 191725 & 204739 & 1342253940,68481 \\
\hline $\mathrm{CO} J=11-10$ & 16384 & 216387 & 215935 & \\
\hline $\operatorname{CO} J=13-12$ & 03925 & 1342191727,8 & 192656 & 1342254900,68195 \\
\hline $\operatorname{CO} J=14-13$ & 03948 & 1342191562,3 & 206501 & \\
\hline $\operatorname{CO} J=15-14$ & 05534 & 1342194732,3 & 216702 & \\
\hline \multirow[t]{2}{*}{$\mathrm{CO} J=16-15$} & 05540 & 1342192673,4 & 206640 & \\
\hline & \multicolumn{4}{|c|}{ PACS } \\
\hline $\mathrm{CO} J=14-13$ through $35-34$ & 1342218570 & 1342218575 & 1342206883 & 1342207774 \\
\hline $\mathrm{CO} J=36-35$ through $48-47$ & 1342218569 & 1342218576 & & 1342207775 \\
\hline
\end{tabular}

Sgr B2(M) is a compact, massive, star-forming core within the central molecular zone of our Galaxy, and its surrounding envelope is an X-ray reflection nebula (Murakami et al. 2000). $\mathrm{W} 49 \mathrm{~N}$ is one of the most luminous and massive star-forming regions within our Galaxy (Galván-Madrid et al. 2013, and references therein), harboring multiple star clusters and tens of O-type stars (Alves \& Homeier 2003; Wu et al. 2016). It is frequently referred to as a starburst region in comparison to the eponymous class of galaxies. Through analysis of the $\mathrm{CO}$ SLEDs in these different well-studied, well-characterized Galactic regions, we aim to empirically interpret extragalactic CO SLEDs.

\section{Observations}

The Herschel observations of EXtra-Ordinary Sources (HEXOS; KPGT_ebergin_1; Bergin et al. 2010) key program includes scans over the full spectral range of HIFI (480-1906 GHz, with gaps from $1280-1430 \mathrm{GHz}$ and $1540-1570 \mathrm{GHz})$ toward Orion $\mathrm{KL}\left[(\alpha, \delta)=\left(05^{\mathrm{h}} 35^{\mathrm{m}} 14^{\mathrm{s}} .3\right.\right.$, $\left.\left.-05^{\circ} 22^{\prime} 33^{\prime \prime} \cdot 7\right)\right]$, Orion South $\left[(\alpha, \delta)=\left(05^{\mathrm{h}} 35^{\mathrm{m}} 13 . \mathrm{s} \cdot 4,-05^{\circ} 24^{\prime}\right.\right.$ $\left.\left.08^{\prime \prime} 1\right)\right]$, the Orion Bar $\left[(\alpha, \delta)=\left(05^{\mathrm{h}} 35^{\mathrm{m}} 20^{\mathrm{s}} 6,-05^{\circ} 25^{\prime} 14^{\prime \prime} 0\right)\right]$, Sgr B2(M) $\left[(\alpha, \delta)=\left(17^{\mathrm{h}} 47^{\mathrm{m}} 20^{\mathrm{s}} 35,-28^{\circ} 23^{\prime} 03^{\prime \prime} 0\right)\right]$, and Sgr B2(N) $\left[(\alpha, \delta)=\left(17^{\mathrm{h}} 47^{\mathrm{m}} 19^{\mathrm{s}} .88,-28^{\circ} 22^{\prime} 18^{\prime \prime} 4\right)\right]$. Targets were observed in dual beam switch (DBS) spectral scan mode with reference positions offset by $3^{\prime}$, and the wide band spectrometer (WBS) was employed to provide $1.1 \mathrm{MHz}$ resolution. In all sources, the HIFI spectra cover rotational transitions of ${ }^{12} \mathrm{C}^{16} \mathrm{O}$ (hereafter referred to simply as $\mathrm{CO}$ ) out of the $5 \leqslant J_{u} \leqslant 16$ levels. ${ }^{8}$ Toward Orion KL, however, the HIFI beam does not encompass the entire emitting region for $J_{u} \geqslant 13$, so for these transitions there are separate pointings toward the hot core $\left[(\alpha, \delta)=\left(05^{\mathrm{h}} 35^{\mathrm{m}} 14.5,-05^{\circ} 22^{\prime} 30.9\right)\right]$ and compact ridge $\left[(\alpha, \delta)=\left(05^{\mathrm{h}} 35^{\mathrm{m}} 14.1,-05^{\circ} 22^{\prime} 36^{\prime \prime} .5\right)\right]$ components (Crockett et al. 2014). Individual analyses of the full spectra have been reported for Orion KL (Crockett et al. 2014), Orion $S$ (Tahani 2013; Tahani et al. 2016), the Orion Bar (Nagy et al. 2017), and Sgr B2(N) (Neill et al. 2014).

\footnotetext{
The $J=12-11$ transition falls in the gap in frequency coverage.
}

Unlike the full spectral scans made of Sgr B2 and Orion sources, HIFI observations of W49N $\left[(\alpha, \delta)=\left(19^{\mathrm{h}} 10^{\mathrm{m}} 13^{\mathrm{s}} .2\right.\right.$, $\left.09^{\circ} 06^{\prime} 12^{\prime \prime} 0\right)$ ] were made in targeted spectral windows as part of multiple science and calibration programs. In total, five $\mathrm{CO}$ transitions in the HIFI frequency range were observed toward W49N. CO $J=6-5$ was covered by the PRISMAS (PRobing InterStellar Molecules with Absorption line Studies; KPGT_mgerin_1) key program in observations targeting $\mathrm{D}_{2} \mathrm{H}^{+}$at $692 \mathrm{GHz}, J=7-6$ by OT1_mgerin_4 in observations targeting $\mathrm{C}$ I at $809 \mathrm{GHz}$, and $J=\overline{8}-7$ by OT1_cvastel_2 in observations targeting HDO at $919 \mathrm{GHz}$. Observations of these three transitions utilized the WBS, and were made in DBS mode. On the fly (OTF) maps of CO $J=10-9$ and $J=13-12$ were made as part of calibration observations. Table 1 lists the ObsIDs that contain $\mathrm{CO}$ transitions for each source.

Sgr B2, Orion KL, the Orion Bar, and Orion S were also observed as part of the HEXOS program with the PACS spectrometer over the $\sim 54-190 \mu \mathrm{m}$ spectral range, covering the $14 \leqslant J_{u} \leqslant 48$ rotational transitions of $\mathrm{CO}{ }^{9}$ PACS observations of the Orion Bar will be presented by C. Joblin et al. (in preparation), so we do not discuss them further. The PRISMAS key program included PACS observations of W49N. The PACS spectrometer (Poglitsch et al. 2010) provides 25 spectra over a $47^{\prime \prime} \times 47^{\prime \prime}$ field of view resolved in $5 \times 5$ spatial pixels ("spaxels"), each with an angular size of 9 ". $4 \times 9$ ". 4 on the sky. The resolving power of the grating spectrometer varies between $R \sim 1000-1500(\sim 108-190 \mu \mathrm{m}$ range), $R \sim 1700-3000$ (70-94 $\mu \mathrm{m}$ range), and $R \sim$ 2700-5500 (54-70 $\mu \mathrm{m}$ range). Spectra for all sources were obtained in the pointed Range Spectroscopy SED mode. Orion $\mathrm{S}$ and W49N were observed in the standard "chop-nod" mode with a chopper throw of \pm 6 arcmin. Owing to the very high far-infrared continuum fluxes toward Sgr B2(M) and Orion KL (above the nominal saturation limits of PACS), these sources were observed in a specific non-standard engineering procedure (PacsCalWaveCalNo-ChopBurst; see Goicoechea et al. 2015). In order to avoid contamination from the bright Orion and $\mathrm{Sgr}$ B2 extended clouds, the "unchopped" observing mode was

\footnotetext{
9 Some wavelength ranges were affected by low spectral response (98-102 $\mu \mathrm{m})$ and spectral leakage (see the PACS observer's manual at http://herschel.esac.esa.int/Docs/PACS/html/pacs_om.html). We do not consider $\mathrm{CO}$ lines lying in these ranges.
} 
used. In this mode, background subtraction is achieved by removing the telescope spectrum measured on a distant reference OFF-position (in our case separated by $\sim 20$ arcmin). ObsIDs corresponding to these data are also shown in Table 1. Herein, we focus our analysis solely on the $\mathrm{CO}$ rotational transitions within the PACS and HIFI spectral scans.

\section{Data Reduction}

\subsection{HIFI}

As mentioned in Section 2, HIFI observations of $\mathrm{CO}$ in W49N are comprised of data from multiple programs, such that our data set is not uniform. Single pointing observations of the $J=6-5$ and $J=7-6$ transitions were processed to Level 2 using the standard Herschel Interactive Processing Environment (HIPE; Ott 2010) pipeline v12.0, and those of the $J=8-7$ transition were processed to Level 2 using HIPE v13.0. Baselines were subtracted using a first order polynomial and spectra taken at different local oscillator frequencies were averaged together. Despite the beams for the $\mathrm{H}$ and $\mathrm{V}$ polarizations being separated by a few arcseconds, we found no evidence for sharp discrepancies between their associated spectra, so the two polarizations are also averaged together. OTF maps of the $J=10-9$ and $J=13-12$ transitions were processed to Level 2 via the standard HIPE pipeline v14.0 and converted to CLASS format. No spectrum in the OTF maps was taken at the same position as the single pointing observations. To extract a single spectrum at this position we take a weighted average of the spectra within the OTF maps. Individual spectra are weighted by $\exp \left(-r^{2} / \theta^{2}\right)$, where $r$ is the angular separation between each observation and the single pointing position and $\theta$ is the radius of the HIFI beam (i.e., Gaussian half-width at half maximum) at the transition frequency.

The full HIFI spectral scans of Orion and Sgr B2 sources were processed using the same methods described in Neill et al. (2014). Orion $\mathrm{S}$ was processed through HIPE v9.0 (Tahani 2013), Orion KL through HIPE v10.3, and Sgr B2 (M) through HIPE v8.0. These reduced data products are available for download via the Herschel Science Archive as user provided data products. ${ }^{10}$ All spectra were rescaled to account for the most up-to-date main beam efficiencies. ${ }^{11}$ Resulting $\mathrm{CO}$ emission lines are shown in Figure 1.

\section{2. $P A C S$}

PACS data were also processed using HIPE. Pointed observations with the PACS array do not provide fully spatially sampled maps. In particular, the individual spaxels do not fill the spectrometer point-spread function (PSF) entirely. The measured width of the PSF is relatively constant for $\lambda \lesssim 100 \mu \mathrm{m}$ (about the spaxel angular size) but increases at longer wavelengths. About 74\% (41\%) of the emission from a point source would fall in a given spaxel at about $54 \mu \mathrm{m}$ $(190 \mu \mathrm{m})$. For sources with semi-extended emission this means that accurate line fluxes can only be extracted by adding the fluxes measured in apertures that cover several spaxels. Orion KL and Sgr B2 data were calibrated and reduced as

\footnotetext{
${ }^{10}$ http://www.cosmos.esa.int/web/herschel/user-provided-data-products

11 The HIFI Beam: Release \#1; document ID HIFI-ICC-RP-2014-001 (Mueller et al.) http://herschel.esac.esa.int/twiki/pub/Public/ HifiCalibrationWeb/HifiBeamReleaseNote_Sep2014.pdf Table 2 and Equation (8), therein.
}

described in Goicoechea et al. (2015). Reductions of Orion S and W49N observations follow a similar method to that described in Gerin et al. (2015).

\section{Analysis}

\subsection{PACS Data}

After data reduction and line identification, a polynomial baseline was subtracted in a narrow wavelength window around each detected CO line (with $J_{u} \geqslant 14$ ). Line fluxes (in $\mathrm{W} \mathrm{m}^{-2}$ ) were extracted by fitting Gaussians to every line detected in every spaxel. Total line fluxes (within a given aperture) were obtained by summing the line fluxes measured in the different individual spaxels. In most cases we added the fluxes from all 25 spaxels within the $5 \times 5$ PACS array. However, fluxes in Orion KL were determined as described in Goicoechea et al. (2015) for a $3 \times 3$ spaxel $\left(\approx 30^{\prime \prime} \times 30^{\prime \prime}\right)$ aperture centered on the hot core. This smaller region was used for extracting fluxes instead of the full array since the PACS footprint centered on Orion KL is contaminated by Orion $\mathrm{H}_{2}$ Peak 1 , which gives rise to most of the high- $J \mathrm{CO}$ emission (see Figure 1 in Goicoechea et al. 2015). The line fluxes toward Sgr B2(M) and Orion $S$ were extracted from the full $5 \times 5$ spaxel aperture. In $\mathrm{W} 49 \mathrm{~N}$ we extracted $\mathrm{CO}$ line fluxes both from the central spaxel alone with a point-source correction applied, and from the full $5 \times 5$ array. Our reasons for testing both methods are discussed in Section 4.3. The PACS flux calibration accuracy is limited by detector response drifts and slight pointing offsets, and the absolute flux calibration accuracy is estimated to be on the order of $30 \% .^{12}$ $\mathrm{CO}$ line fluxes determined from our analysis are reported in Table 2 .

\subsection{HIFI Data}

Our analysis of the CO $5 \leqslant J_{u} \leqslant 16$ emission lines is performed using the spectra generated from the reduction described in Section 3.1. Integrated line fluxes $\left(\int T_{\mathrm{MB}} d v\right)$ in units of $\mathrm{K} \mathrm{km} \mathrm{s}^{-1}$ are extracted from the spectra in Figure 1 as described below, and are converted to intensity and flux via

$$
\begin{aligned}
\frac{\text { Intensity }}{\left(\mathrm{W} \mathrm{m}^{-2} \mathrm{sr}^{-1}\right)} & =\frac{\nu^{3} 2 k_{\mathrm{b}}}{c^{3}} \int T_{\mathrm{MB}} d v \\
& =1.0248 \times 10^{-18}\left(\frac{\nu}{\mathrm{GHz}}\right)^{3}\left(\frac{\int T_{\mathrm{MB}} d v}{\mathrm{~K} \mathrm{~km} \mathrm{~s}^{-1}}\right)
\end{aligned}
$$

and

$$
\begin{aligned}
\frac{\text { Flux }}{\left(\mathrm{W} \mathrm{m}^{-2}\right)} & =\frac{\nu^{3} 2 k_{\mathrm{b}} \Omega}{c^{3}} \int T_{\mathrm{MB}} d v \\
& =1.0248 \times 10^{-18}\left(\frac{\Omega}{\mathrm{sr}}\right)\left(\frac{\nu}{\mathrm{GHz}}\right)^{3}\left(\frac{\int T_{\mathrm{MB}} d v}{\mathrm{~K} \mathrm{~km} \mathrm{~s}^{-1}}\right)
\end{aligned}
$$

where $\nu$ is the transition frequency, $k_{\mathrm{b}}$ is the Boltzmann constant, $c$ is the speed of light, and $\Omega$ is the main beam solid angle. Line fluxes are reported in Table 2, along with $\Omega$ at the pertinent frequencies.

\footnotetext{
$\overline{12}$ PACS Spectroscopy performance and calibration, PACS/ICC document ID PICC-KL-TN-041 (Vandenbussche et al.).
} 

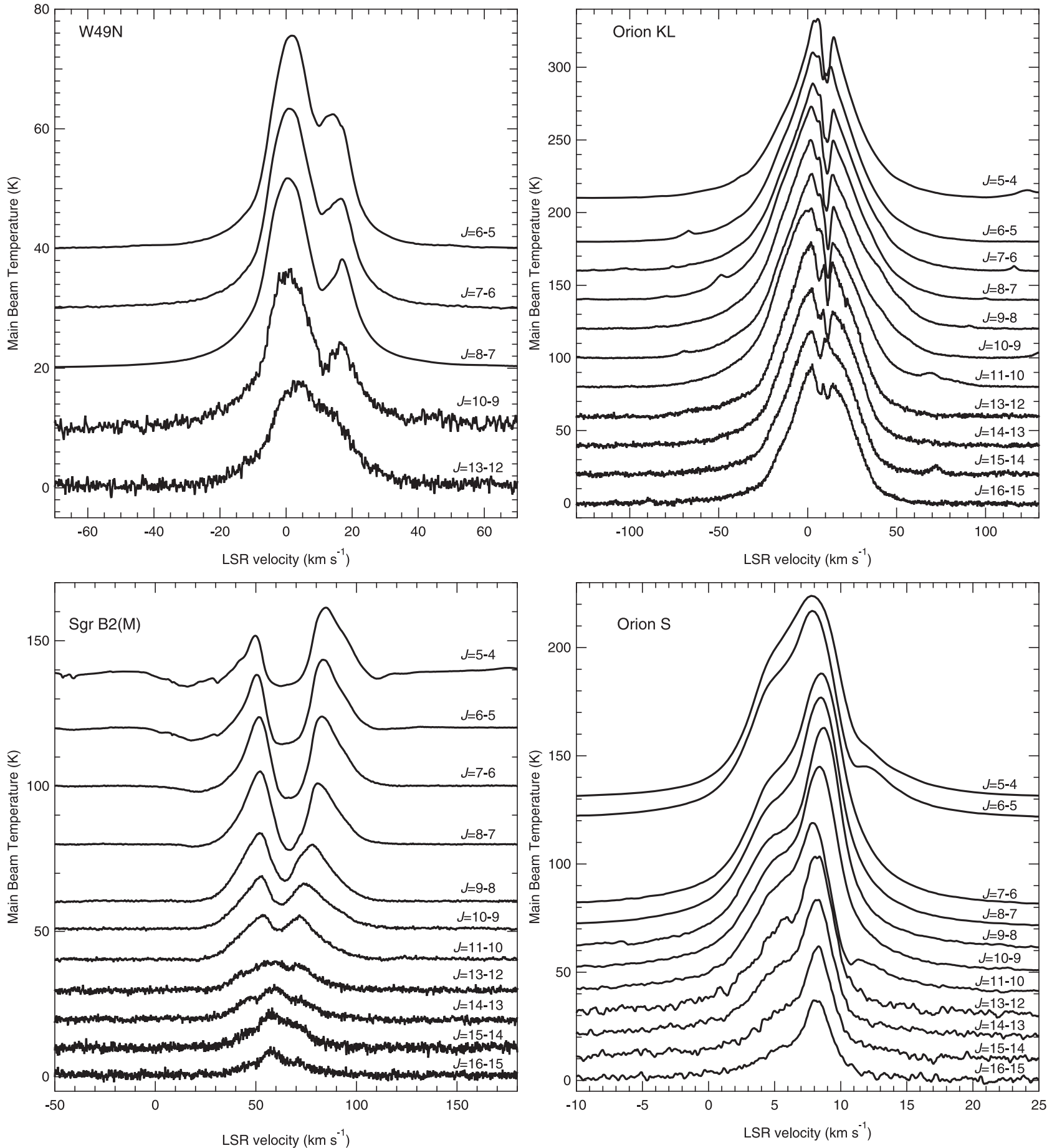

Figure 1. CO emission lines in W49N (top left), Orion KL (top right), Sgr B2(M) (bottom left) and Orion S (bottom right). Note the different velocity and temperature scales in each panel. Spectra are shifted vertically for clarity. In Orion KL the spectra displayed for the $13 \leqslant J_{u} \leqslant 16$ transitions are from the observations targeting the compact ridge.

\subsubsection{W49N}

$\mathrm{CO}$ emission lines in W49N are shown in the top left panel of Figure 1. They display the same double peaked profiles as observed in $\mathrm{HCN}$ and $\mathrm{HCO}^{+}$which are caused by strong self absorption and interpreted as a signature of gas infall (Roberts et al. 2011). The self-absorption scenario is favored over two separate emission components because the weaker isotopologue emission (e.g., $\mathrm{H}^{13} \mathrm{CO}^{+}, \mathrm{H}^{13} \mathrm{CN}$ ) is singly peaked at about $7 \mathrm{~km} \mathrm{~s}^{-1}$, the systemic velocity of W49N. We determine integrated line fluxes for $\mathrm{CO}$ over the velocity interval from -40 to $45 \mathrm{~km} \mathrm{~s}^{-1}$.

\subsubsection{Orion $K L$}

The top right panel of Figure 1 displays the $\mathrm{CO}$ emission lines observed toward Orion KL. The broad line profiles likely contain emission from the various well-known components such as the hot core, compact ridge, and plateau (high-velocity and low-velocity outflows), with the extended ridge giving rise 
Table 2

CO Line Fluxes

\begin{tabular}{|c|c|c|c|c|c|}
\hline \multirow[b]{3}{*}{ Transition } & \multirow{3}{*}{$\begin{array}{c}\Omega \\
\left(10^{-9} \mathrm{sr}\right)\end{array}$} & Orion $\mathrm{S}$ & Orion $\mathrm{KL}$ & Sgr B2(M) & W49N \\
\hline & & \multicolumn{4}{|c|}{ Flux } \\
\hline & & $\left(10^{-15} \mathrm{~W} \mathrm{~m}^{-2}\right)$ & $\left(10^{-15} \mathrm{~W} \mathrm{~m}^{-2}\right)$ & $\left(10^{-15} \mathrm{~W} \mathrm{~m}^{-2}\right)$ & $\left(10^{-15} \mathrm{~W} \mathrm{~m}^{-2}\right)$ \\
\hline \multicolumn{6}{|l|}{$\overline{\mathrm{HIFI}}$} \\
\hline$J=5-4$ & 24.0 & $3.53 \pm 0.05$ & $26.2 \pm 0.3$ & $1.37 \pm 0.03$ & $\ldots$ \\
\hline$J=6-5$ & 17.1 & $4.59 \pm 0.06$ & $37.5 \pm 0.4$ & $2.54 \pm 0.01$ & $4.80 \pm 0.24$ \\
\hline$J=7-6$ & 12.3 & $5.61 \pm 0.04$ & $41.8 \pm 0.5$ & $5.16 \pm 0.02$ & $5.16 \pm 0.26$ \\
\hline$J=8-7$ & 9.44 & $6.17 \pm 0.04$ & $51.5 \pm 0.5$ & $6.05 \pm 0.16$ & $5.86 \pm 0.29$ \\
\hline$J=9-8$ & 7.52 & $6.37 \pm 0.05$ & $57.9 \pm 0.6$ & $7.74 \pm 0.11$ & $\ldots$ \\
\hline$J=10-9$ & 6.64 & $7.12 \pm 0.04$ & $66.1 \pm 0.7$ & $8.08 \pm 0.21$ & $6.79 \pm 0.34$ \\
\hline$J=11-10$ & 5.49 & $6.51 \pm 0.05$ & $74.3 \pm 0.8$ & $7.23 \pm 0.16$ & $\ldots$ \\
\hline$J=12-11$ & $\ldots$ & $\ldots$ & $\ldots$ & $\ldots$ & $\ldots$ \\
\hline$J=13-12$ & 3.59 & $5.94 \pm 0.09$ & $67.9 \pm 0.9$ & $4.82 \pm 0.14$ & $5.43 \pm 0.27$ \\
\hline$J=14-13$ & 3.09 & $5.25 \pm 0.07$ & $66.1 \pm 1.0$ & $5.11 \pm 0.29$ & $\ldots$ \\
\hline$J=15-14$ & 2.78 & $4.25 \pm 0.08$ & $65.4 \pm 1.1$ & $5.25 \pm 0.10$ & $\ldots$ \\
\hline$J=16-15$ & 2.45 & $2.97 \pm 0.07$ & $61.2 \pm 1.1$ & $3.24 \pm 0.14$ & $\ldots$ \\
\hline PACS & & $5 \times 5$ & $3 \times 3$ & $5 \times 5$ & $5 \times 5$ \\
\hline$J=14-13$ & $\ldots$ & 41.58 & 230.3 & 18.00 & 17.60 \\
\hline$J=15-14$ & $\ldots$ & 37.93 & 253.9 & 15.28 & 18.30 \\
\hline$J=16-15$ & $\ldots$ & 33.89 & 294.2 & 11.41 & 16.00 \\
\hline$J=17-16$ & $\ldots$ & 27.78 & 323.0 & $\ldots$ & 13.90 \\
\hline$J=18-17$ & $\ldots$ & 21.91 & 269.0 & $\cdots$ & 11.10 \\
\hline$J=19-18$ & $\ldots$ & 15.34 & 318.4 & $\ldots$ & 12.60 \\
\hline$J=20-19$ & $\ldots$ & 12.65 & 317.5 & $\ldots$ & 11.50 \\
\hline$J=21-20$ & $\ldots$ & 9.23 & 263.4 & $\ldots$ & 9.23 \\
\hline$J=22-21$ & $\ldots$ & 5.65 & 233.9 & $\ldots$ & 7.73 \\
\hline$J=23-22$ & $\ldots$ & 14.97 & $\ldots$ & $\ldots$ & 5.76 \\
\hline$J=24-23$ & $\ldots$ & 3.99 & 156.4 & $\ldots$ & 4.95 \\
\hline$J=25-24$ & $\ldots$ & 3.70 & $\ldots$ & $\ldots$ & 3.26 \\
\hline$J=26-25$ & $\ldots$ & $\ldots$ & $\ldots$ & $\ldots$ & $\ldots$ \\
\hline$J=27-26$ & $\ldots$ & $\ldots$ & $\ldots$ & $\ldots$ & 2.20 \\
\hline$J=28-27$ & $\ldots$ & 2.74 & 56.5 & $\ldots$ & 2.22 \\
\hline$J=29-28$ & $\ldots$ & 2.04 & 32.9 & $\ldots$ & 1.91 \\
\hline$J=30-29$ & $\ldots$ & 1.90 & 32.9 & $\ldots$ & 1.14 \\
\hline$J=31-30$ & $\ldots$ & $\ldots$ & $\ldots$ & $\ldots$ & $\ldots$ \\
\hline$J=32-31$ & $\ldots$ & $\ldots$ & 22.2 & $\ldots$ & 0.86 \\
\hline$J=33-32$ & $\ldots$ & $\ldots$ & 20.1 & $\ldots$ & 0.36 \\
\hline$J=34-33$ & $\ldots$ & $\ldots$ & 12.7 & $\ldots$ & 0.34 \\
\hline$J=35-34$ & $\cdots$ & $\cdots$ & 7.0 & $\cdots$ & $\ldots$ \\
\hline$J=36-35$ & $\ldots$ & $\ldots$ & 5.0 & $\ldots$ & $\ldots$ \\
\hline$J=37-36$ & $\ldots$ & $\ldots$ & 3.1 & $\ldots$ & $\ldots$ \\
\hline$J=38-37$ & $\ldots$ & $\ldots$ & 4.2 & $\ldots$ & $\ldots$ \\
\hline$J=39-38$ & $\ldots$ & $\ldots$ & 2.2 & $\ldots$ & $\ldots$ \\
\hline$J=40-39$ & $\ldots$ & $\ldots$ & 1.3 & $\ldots$ & $\ldots$ \\
\hline$J=41-40$ & $\ldots$ & $\cdots$ & 0.9 & $\cdots$ & $\ldots$ \\
\hline$J=42-41$ & $\ldots$ & $\ldots$ & 0.8 & $\ldots$ & $\ldots$ \\
\hline
\end{tabular}

Note. Absolute flux calibration accuracy is estimated to be about $30 \%$ for PACS.

to some of the self absorption near $10 \mathrm{~km} \mathrm{~s}^{-1}$ (Blake et al. 1987; Crockett et al. 2014). Integrated line fluxes were determined over the velocity interval from -80 to $90 \mathrm{~km} \mathrm{~s}^{-1}$. Reported uncertainties account for the rms noise level in the spectra (measured from -125 to $-100 \mathrm{~km} \mathrm{~s}^{-1}$ ) and the weak emission features caused by other species that are within the velocity interval over which we measure integrated fluxes. Note that all of the weak emission features seen in the Orion KL spectra at various LSR velocities are caused by molecules other than $\mathrm{CO}$ (e.g., $\mathrm{SO}_{2}$ and $\mathrm{CH}_{3} \mathrm{OH}$ ). The emitting species have been identified and modeled by Crockett et al. (2014), and like the aforementioned reduced spectral scans, those models are also available as user provided data products.

\subsection{3. $\mathrm{Sgr} B 2(M)$}

Toward Sgr B2(M), CO emission is detected from all transitions covered by HIFI. Self absorption caused by the Sgr B2 envelope is strong for transitions with $J_{u} \leqslant 8$, moderately strong for transitions with $9 \leqslant J_{u} \leqslant 11$, and either weak or not present for $J_{u} \geqslant 13$ transitions. Absorption due to gas in the foreground spiral arms is also seen for $J_{u} \leqslant 8$ transitions at velocities blueshifted from $63 \mathrm{~km} \mathrm{~s}^{-1}$, the 

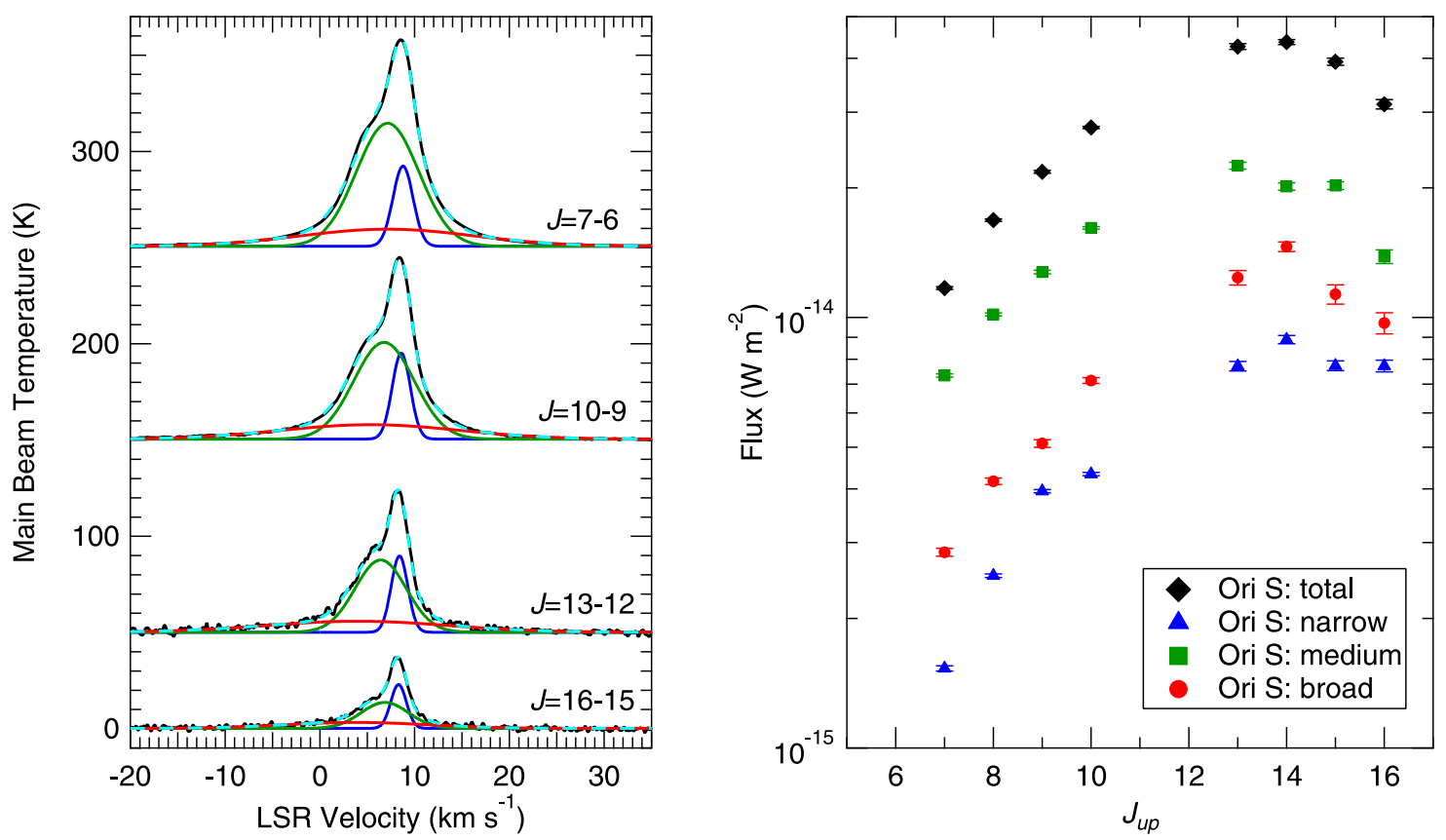

Figure 2. Decomposition of select CO transitions toward Orion S into three Gaussian components is displayed on the left. Spectra are shown in black, with the full fit given by dashed cyan curves. The narrow PDR component is shown in blue, the shock component in green, and the broad outflow component in red. Fluxes for each of the components - scaled as described in Section 4.3-are plotted on the right side. Color coding matches the fit components in the left panel, although the total flux is marked by black (rather than cyan) diamonds. Flux uncertainties are generally smaller than the plotted markers.

systemic velocity of Sgr B2(M). All of these features are shown in the bottom left panel of Figure 1. These results parallel those found for Sgr B2(N) (Neill et al. 2014). CO line fluxes reported in Table 2 for Sgr B2(M) are integrated over the velocity range $0-130 \mathrm{~km} \mathrm{~s}^{-1}$.

\subsubsection{Orion $S$}

Orion $\mathrm{S}$ is comprised of multiple physical components that can be distinguished via their differing kinematics. $\mathrm{CO}$ emission lines in Orion $\mathrm{S}$ are shown in the bottom right panel of Figure 1 , and they display a narrow $\left(\mathrm{FWHM} \approx 2 \mathrm{~km} \mathrm{~s}^{-1}\right.$ ) component centered at about $8.5 \mathrm{~km} \mathrm{~s}^{-1}$, a medium $\left(\right.$ FWHM $\approx 7 \mathrm{~km} \mathrm{~s}^{-1}$ ) component centered at about $7 \mathrm{~km} \mathrm{~s}^{-1}$, and a broad (FWHM $\approx 20 \mathrm{~km} \mathrm{~s}^{-1}$ ) component centered at about $5 \mathrm{~km} \mathrm{~s}^{-1}$. This structure mimics that of $\mathrm{H}_{2} \mathrm{O}$ emission observed toward both low and high-mass protostars (e.g., Kristensen et al. 2012; San José-García et al. 2016). We fit these three components with Gaussian functions, and our decomposition of the $\mathrm{CO}$ emission for select transitions is shown in left-hand side of Figure 2. The profile of the narrow component at $8.5 \mathrm{~km} \mathrm{~s}^{-1}$ is similar to that of $\mathrm{CO}$ emission in the Orion Bar (Nagy et al. 2013), and likely arises from the portion of Orion $S$ that is being illuminated by the Trapezium stars, i.e., in a PDR. The medium component accounts for most of the line flux and is likely due to shocks associated with protostellar activity within the region. The broad component likely corresponds to the outflows detected in $\mathrm{SiO}$ (Ziurys et al. 1990) and CO (Schmid-Burgk et al. 1990). A fourth component is visible in Figure 3 as weak emission $(<1 \mathrm{~K})$ extending to high velocities $\left( \pm 80 \mathrm{~km} \mathrm{~s}^{-1}\right)$, and is detected for all transitions with $J_{u} \leqslant 11$. This emission corresponds to the highly collimated outflows observed in $\mathrm{CO} J=2-1$ by Zapata et al. (2005). It is not detected in the $J_{u} \geqslant 13$ transitions simply because the HIFI beam at these frequencies no longer encompasses any portion of the collimated outflows.

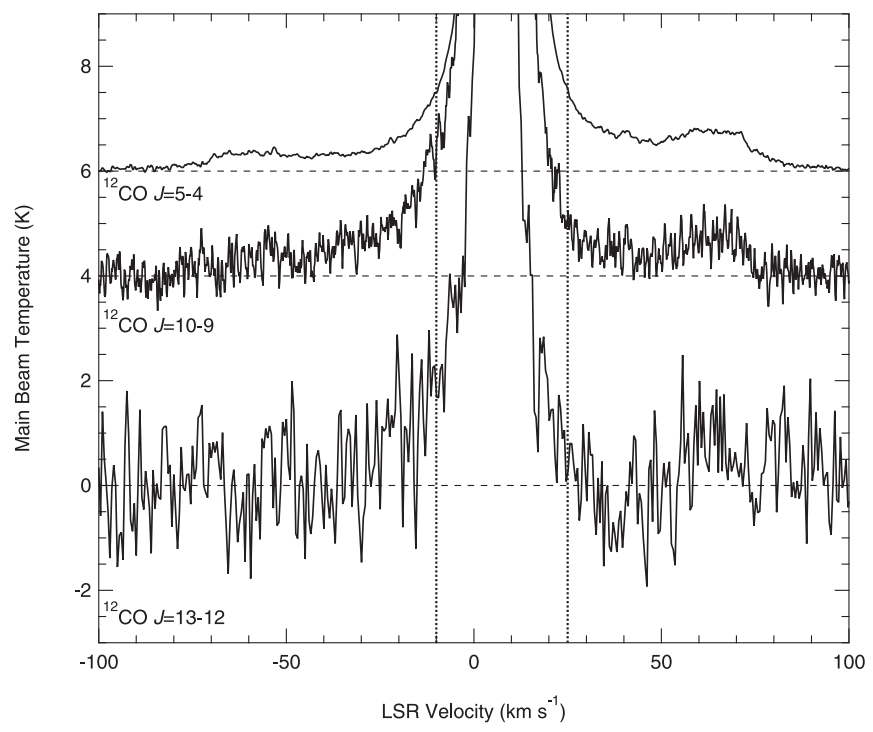

Figure 3. Zoom in on select $\mathrm{CO}$ transitions toward Ori S showing $\mathrm{CO}$ "bullets." Spectra have been shifted vertically for clarity, and the zero level for each spectrum is marked by a horizontal dashed line. The $J=13-12$ spectrum has been smoothed to $0.5 \mathrm{~km} \mathrm{~s}^{-1}$ resolution. Vertical dotted lines denote the full velocity range shown in the bottom right panel of Figure 1.

Careful inspection of the $\mathrm{CO}$ emission lines from Orion $\mathrm{S}$ in Figure 1 reveals that the $J=5-4,6-5$, and 11-10 profiles peak at velocities about $0.5 \mathrm{~km} \mathrm{~s}^{-1}$ lower than the other transitions, and show what appear as small absorption features at about $11 \mathrm{~km} \mathrm{~s}^{-1}$. These artifacts are due to imperfect removal of $\mathrm{CO}$ emission that was in the reference beam at the off position (Tahani et al. 2016). As a result, the fit parameters for the different Gaussian components (i.e., $v_{\mathrm{LSR}}$, FWHM, and $\int T d v$ ) found for these transitions do not agree with those found for the unaffected transitions, and we exclude them from the remainder 

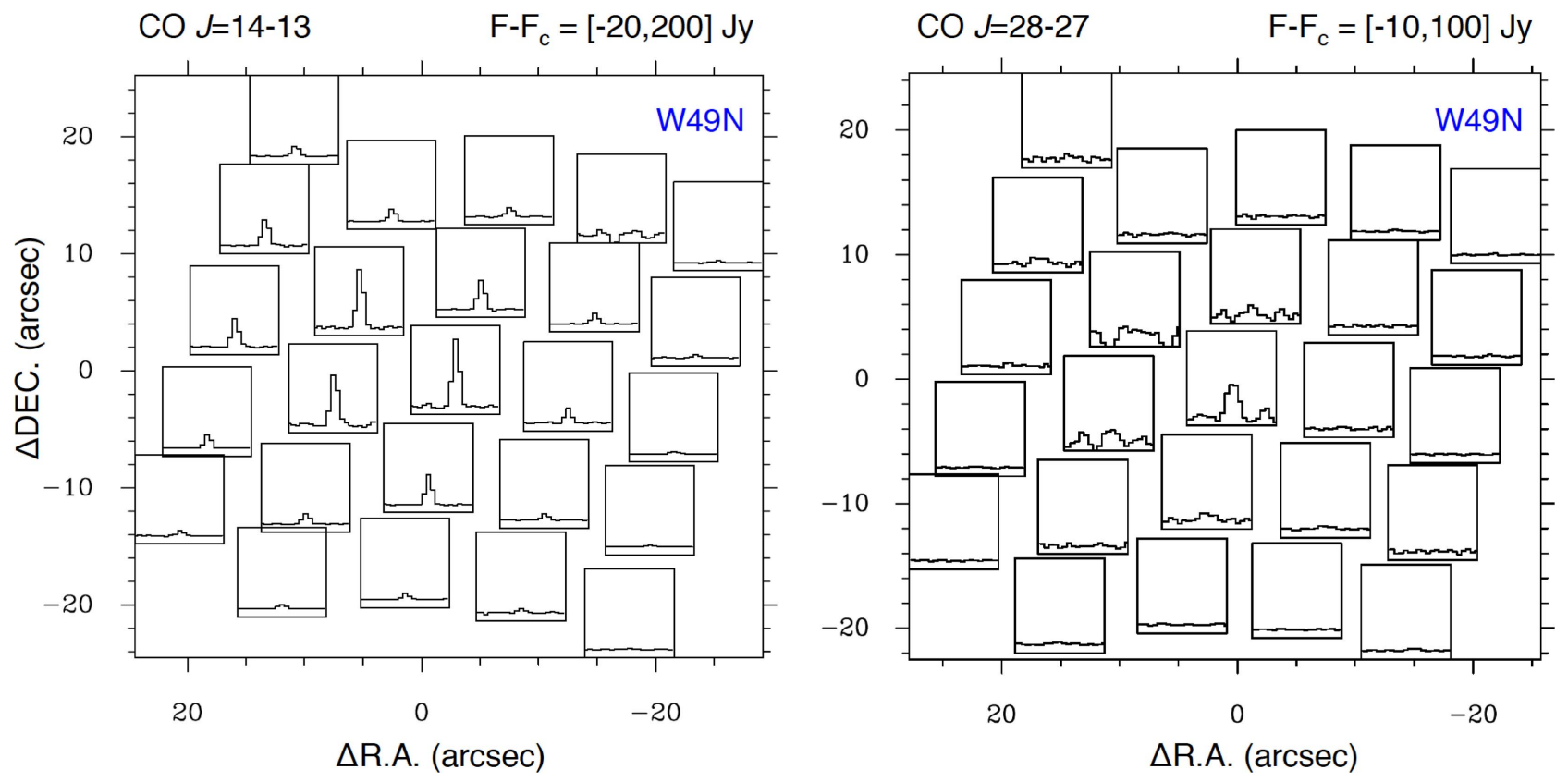

Figure 4. Continuum-subtracted high- $J$ CO maps for W49N obtained with the PACS array in 25 spaxels. The line flux scale (in Jy) is indicated in the top right of each panel. The $(0,0)$ position corresponds to $(\alpha, \delta)=\left(19^{\mathrm{h}} 10^{\mathrm{m}} 13^{\mathrm{s}} .1,09^{\circ} 06^{\prime} 12^{\prime \prime} 0\right)$.

of our analysis. Using our fits to the $\mathrm{CO}$ emission lines we can generate SLEDs for each of the three different components as shown in the right side of Figure 2. All three components show roughly the same overall shape, with SLEDs peaking around $J_{u}=14$.

\subsection{Combining PACS and HIFI Results}

For Orion S, Orion KL, and Sgr B2(M) we have determined fluxes for the $J_{u}=14,15$, and 16 transitions from both HIFI and PACS observations. As can be seen in Table 2, the values determined from the two different instruments do not agree. To generate a CO SLED without discontinuities, we scale HIFI fluxes given the following reasoning. In the limit of an unresolved point source, the flux measured is independent of the beam size. In the limit of a resolved source with uniform emission, the intensity measured is independent of beam size. By comparing PACS fluxes to HIFI fluxes and PACS intensities to HIFI intensities, we can determine which scenario is more likely applicable for each source. For Orion $\mathrm{S}$, the PACS fluxes reported in Table 2 come from the full $5 \times 5$ spaxel array $(47 \times 47 \mathrm{arcsec})$. Fluxes for the $J_{u}=14,15$, and 16 transitions determined from HIFI are lower than those determined from PACS, while intensities determined from HIFI are higher than those determined from PACS. For all three transitions, a roughly constant scaling factor $(6 \%$ variation) can be used to convert PACS intensities to HIFI intensities, but not to convert PACS fluxes to HIFI fluxes ( $\sim 50 \%$ variation). This potentially indicates a source size that is larger than the HIFI beam(s), but smaller than the PACS $5 \times 5$ footprint, a scenario confirmed by inspection of the mid$J \mathrm{CO}$ emission lines observed in each individual spaxel toward Orion S. If so, then the fluxes measured by PACS are correct, while those measured by HIFI are too low. Similarly, the intensities measured by HIFI are correct, while those measured by PACS are underestimated. Dividing the PACS flux by the HIFI intensity gives the source size (assuming uniform emission), and this can be used to scale the HIFI fluxes to the values that would have been measured had the beams fully encompassed the emitting region. In this way, we remove the discrepancies between the PACS and HIFI fluxes reported in Table 2, which would otherwise appear as discontinuities in the various CO SLEDs. The scaling factors used in this conversion for each source are $2.58 \times 10^{-8} / \Omega$ for Orion $\mathrm{S}, 1.11 \times 10^{-8} /$ $\Omega$ for Orion KL, and $9.21 \times 10^{-9} / \Omega$ for Sgr B2(M), where $\Omega$ is the beam solid angle at the transition frequency as reported in Table 2.

For W49N no CO transition was observed by both PACS and HIFI. Examination of $\mathrm{CO}$ spectra observed with each PACS spaxel reveals that the emitting region changes as a function of upper state energy. The lowest lines show emission over multiple spaxels, while the highest lines are concentrated in only the central spaxel. This is demonstrated in Figure 4 where the $J=14-13$ and $J=28-27$ spectra in each PACS spaxel show differences in the emitting region. Because of the changing source size, $\mathrm{CO}$ line fluxes extracted from the full $5 \times 5$ array are larger than those extracted from the central spaxel alone with a point-source correction applied, except at high $J_{u}$ where the emission becomes concentrated. The HIFI beams for the observed $\mathrm{CO}$ transitions are larger than 1 spaxel, smaller than the full PACS array, and also smaller than the CO emitting region seen in the HIFI OTF integrated intensity maps, further complicating the analysis. We choose to use the $5 \times 5$ PACS fluxes throughout the remainder of our analysis in order to avoid "throwing away" flux from the mid- $J$ CO lines. HIFI fluxes are scaled by $1.23 \times 10^{-8} / \Omega$ to remove the discontinuity between PACS and HIFI fluxes. All of these issues highlight the difficulties inherent in combining emission line fluxes extracted from detectors with different beam sizes covering different portions of a target region that itself changes size and shape as a function of transition energy.

\section{Discussion}

The CO SLEDs resulting from the analysis described above are shown in the top panel of Figure 5 for Sgr B2(M), 

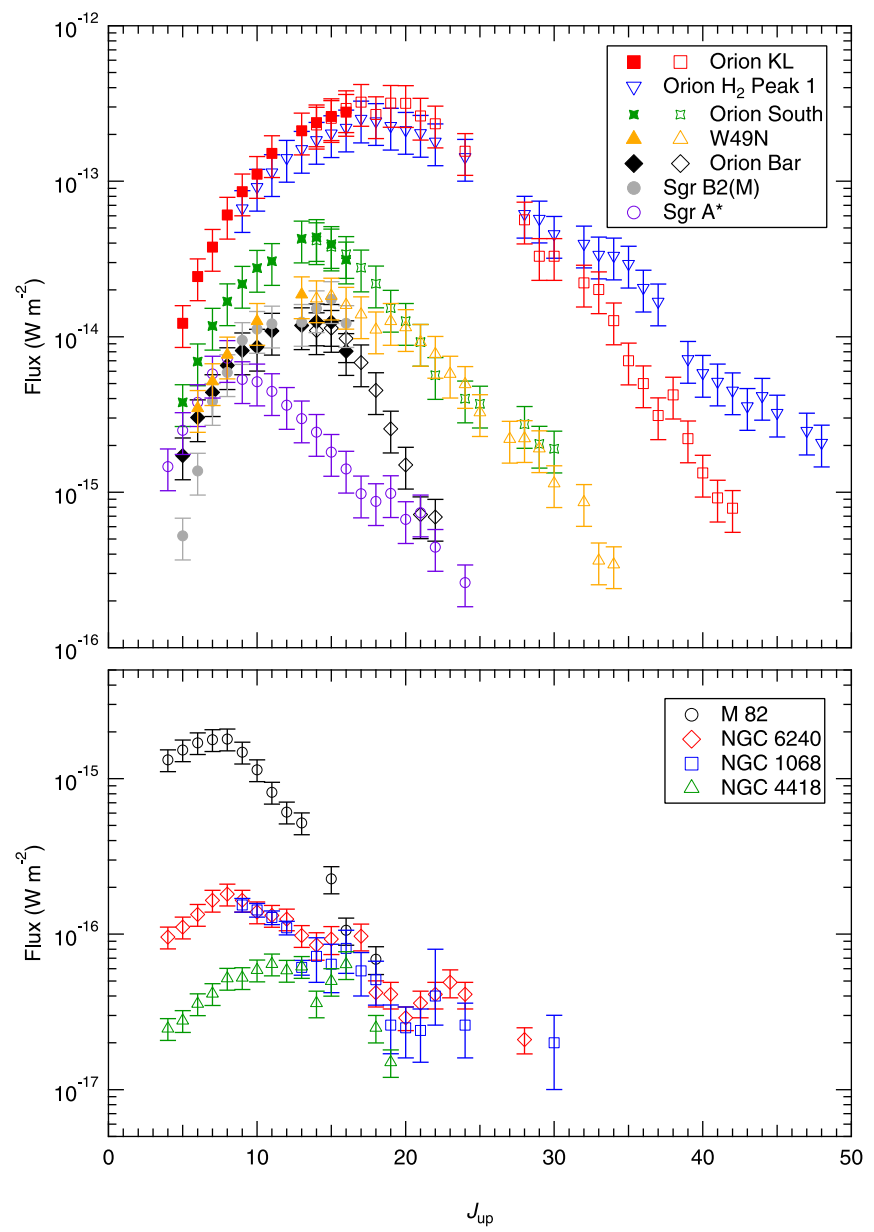

Figure 5. CO spectral line energy distributions for Galactic sources (top), and for extragalactic sources (bottom). Filled symbols denote fluxes determined from HIFI observations, and open symbols from either SPIRE $\left(J_{u} \leqslant 13\right)$ or PACS $\left(J_{u} \geqslant 14\right)$ observations. The $30 \%$ uncertainties in PACS fluxes have been applied to the HIFI fluxes as well given our scaling procedure. References for published line fluxes are as follows: Orion $\mathrm{H}_{2}$ Peak 1 (Goicoechea et al. 2015); Orion Bar (C. Joblin et al. 2017, in preparation),; Sgr A* (Goicoechea et al. 2013); NGC 1068 (Hailey-Dunsheath et al. 2012; Spinoglio et al. 2012; Janssen et al. 2015); NGC 6240 (Mashian et al. 2015; Rosenberg et al. 2015); M 82 (Kamenetzky et al. 2012; Mashian et al. 2015); NGC 4418 (Mashian et al. 2015; Rosenberg et al. 2015).

Orion KL, Orion S, and W49N. Additionally, the top panel displays CO SLEDs in the Galactic sources Orion $\mathrm{H}_{2}$ Peak 1 (Goicoechea et al. 2015), the Orion Bar (C. Joblin et al. 2017, in preparation), and Sgr A* (Goicoechea et al. 2013), while the bottom panel shows CO SLEDs from the Seyfert 2 galaxy NGC 1068 (Hailey-Dunsheath et al. 2012; Spinoglio et al. 2012; Janssen et al. 2015), luminous infrared galaxies NGC 6240 (Mashian et al. 2015; Rosenberg et al. 2015) and NGC 4418 (Mashian et al. 2015; Rosenberg et al. 2015), and the starburst galaxy M 82 (Kamenetzky et al. 2012; Mashian et al. 2015). The vertical axes in both the top and bottom panels have been scaled to facilitate direct visual comparison of the CO SLED shapes. We focus first on the CO SLEDs in Galactic sources.

All of the Orion sources are at a distance of about $420 \mathrm{pc}$ (Menten et al. 2007), so differences in those SLEDs are mostly intrinsic to the sources. The Orion Bar shows the simplest profile, and can be considered a template for the CO SLED in a strongly illuminated PDR with $\chi_{\mathrm{UV}} \approx 10^{4}$ (expressed in units of the mean interstellar radiation field from Draine 1978). The other Orion sources likely contain PDR components as well, as some portion of the gas is being illuminated by FUV photons from the Trapezium cluster. Indeed, the decomposition of Orion $\mathrm{S}$ line profiles described in Section 4.2.4 shows this PDR component, and demonstrates that it has the smallest contribution to the total emission line flux. Orion $\mathrm{S}$ and Orion $\mathrm{KL}$ are both regions of embedded massive star formation, and the internal energy provided by this process through outflows, shocks, and radiation serves to increase the CO line flux compared to the externally heated Orion Bar. Orion KL has a bolometric luminosity about 10 times that of Orion S (O'Dell et al. 2008, and references therein), hence the larger $\mathrm{CO}$ line fluxes. Simply put, the increasing energy available going from the Orion Bar to Orion $S$ to Orion KL both excites a larger amount of molecular gas and pushes population in the rotational levels of CO to higher $J$, thus producing the observed CO SLEDs. The SLED for Orion $\mathrm{H}_{2}$ Peak $1-$ a region which can be considered a prototypical strong molecular shock-has a different shape with line fluxes decreasing more slowly as $J_{u}$ increases. Excitation in this region is dominated by shock heating as a high-velocity outflow collides with quiescent molecular gas, and emission from the highest $J_{u}$ transitions arises from hot $(T \sim 3000 \mathrm{~K})$, dense $\left(n \sim 10^{7} \mathrm{~cm}^{-3}\right.$ ) gas (Goicoechea et al. 2015).

The Sgr B2(M) and Orion Bar SLEDs are very similar, despite the sources themselves and their $\mathrm{CO}$ emission line profiles being vastly different. As shown in Figure 1 the Sgr B2(M) line profiles are complex and dominated by self absorption from the envelope that surrounds the hot core, whereas the Orion Bar has a single velocity component in emission (see, e.g., Figure 2 in Nagy et al. 2013). Additionally, far-infrared extinction by the Sgr B2 envelope may reduce the observed flux in the $J=16-15$ transition by a factor of 10 below that actually produced by Sgr B2(M) (Etxaluze et al. 2013), and by even larger factors for higher $J_{u}$ lines (hence the non-detections for $J_{u}>16$ transitions). While far-IR extinction only affects certain objects, it is clear that attempting to infer source attributes from the CO line fluxes alone is a complicated and likely degenerate procedure.

The CO SLED in W49N most closely resembles that in Orion S. W49N is one of the most luminous massive starforming regions in our Galaxy-about $10^{3}$ times more luminous than Orion S (Sievers et al. 1991) —and has been considered a template for extragalactic giant $\mathrm{H}$ II regions $(\mathrm{Wu}$ et al. 2016), so this similarity is unexpected. Nagy et al. (2012) concluded that both UV photons and mechanical processes (e.g., winds and outflows) are likely the dominant heating mechanisms in this region, while X-rays do not contribute significantly. It seems likely then that Orion $S$ experiences similar conditions, just on a much smaller scale. Of the Galactic sources shown in Figure 5, the CO SLED in Sgr A* peaks at the lowest value of $J_{u}$. Goicoechea et al. (2013) concluded that UV photons and shocks are responsible for heating the hot molecular gas giving rise to the $\mathrm{CO}$ emission near $\mathrm{Sgr} \mathrm{A}^{*}$, and that presently neither X-rays nor cosmic rays play a large role.

A multitude of galaxies have been observed by Herschel with SPIRE (e.g., Kamenetzky et al. 2014; Rosenberg et al. 2015) and PACS (e.g., Mashian et al. 2015) with CO emission lines as a primary target. We selected the four galaxies shown in the bottom panel of Figure 5 for comparison to our Galactic sources because they present a variety of $\mathrm{CO}$ SLED shapes, including a member of each of the three classes 
defined by Rosenberg et al. (2015). Additionally, NGC 1068 and NGC 6240 have CO emission detected out to the highest $J_{u}$ of any galaxies, providing the most extensive comparisons to Galactic regions, and the CO SLED of NGC 4418 peaks at the highest $J_{u}$ of any galaxy. Note that we have excluded the SPIRE observations of NGC 1068 for $4 \leqslant J_{u} \leqslant 8$ as the larger beam at these frequencies contains two emission regionscircumnuclear disk (CND) and extended ring-whereas the SPIRE $J_{u} \geqslant 9$ and PACS $J_{u} \geqslant 14$ observations only probe the nuclear disk (Hailey-Dunsheath et al. 2012; Spinoglio et al. 2012).

It is immediately apparent that the extragalactic CO SLEDs differ in shape from their Galactic counterparts. In fact, linear combinations of the Orion, W49N, and Sgr B2(M) CO SLEDs are incapable of reproducing those seen in M 82, NGC 1068, and NGC 6240 because all of these Galactic SLEDs peak at higher $J_{u}$ than the extragalactic SLEDs. Even the individual components of Orion S shown in Figure 2 fail in this regard. Only the Sgr A* CO SLED peaks at low enough $J_{u}$ that it could conceivably be used to reconstruct the extragalactic sources, while only the NGC 4418 CO SLED peaks at high enough $J_{u}$ that it could conceivably be reproduced by Galactic sources. These disparities effectively prevent the empirical interpretation of extragalactic CO SLEDs and their underlying excitation mechanisms, yet simultaneously beg the question: Why do most Galactic CO SLEDs not resemble those in other galaxies?

The clearest difference between Galactic and extragalactic CO SLEDs is where the distribution peaks (i.e., which CO emission line has the largest flux). The NGC 4418 CO SLED peaks at $J_{u} \approx 11-13$, the NGC 6240 and M 82 SLEDs at $J_{u}=8$, while the NGC 1068 SLED is increasing toward lower $J_{u}$, with $J_{u}=9$ being the lowest transition where the measured flux arises solely in the CND component. The Orion KL and Orion $\mathrm{H}_{2}$ Peak 1 SLEDs peak near $J_{u}=18$ with emission in Peak 1 extending all the way to $J_{u}=48$, while the Orion Bar, Orion S, W49N, and Sgr B2(M) SLEDs peak near $J_{u}=14$. Sgr A* has a CO SLED that peaks at $J_{u}=8$. Where the CO SLEDs peak depends on the physical conditions of the gas, with hotter, denser gas leading to increased population, and thus flux, for higher $J_{u}$ transitions. While it may at first seem counterintuitive that Galactic star-forming regions appear to harbor more hot, dense molecular gas than regions surrounding active galactic nuclei, this is likely an effect of beam filling factors. At a distance of $14.4 \mathrm{Mpc}$ (Bland-Hawthorn et al. 1997) the 9" $4 \times 9$ "! 4 central spaxel of PACS covers a region about $650 \mathrm{pc}$ on a side in NGC 1068. For NGC 4418 at $d=34 \mathrm{Mpc}$ (Braatz et al. 1997) and NGC 6240 at $d=107 \mathrm{Mpc}$ (Meijerink et al. 2013) the central spaxel covers a region approximately $1.5 \times 1.5 \mathrm{kpc}$ and $4.6 \times 4.6 \mathrm{kpc}$, respectively. $\mathrm{CO}$ line fluxes in $\mathrm{M} 82$ were extracted from the full $47^{\prime \prime} \times 47^{\prime \prime}$ PACS array (Mashian et al. 2015), which covers a region $770 \times 770 \mathrm{pc}$ at $d=3.4 \mathrm{Mpc}$ (Dalcanton et al. 2009). These regions, which do contain hot, dense gas as evidenced by emission from high- $J_{u} \mathrm{CO}$, must also contain large amounts of cooler gas, which emit primarily at lower $J_{u}$ transitions of CO. Emission from the hot, dense gas only fills a small portion of the beam, while emission from the more extended cooler gas fills a much larger portion of the beam and ends up dominating the CO SLED.

Beam filling effects have previously been invoked to describe CO SLEDs observed in the Galactic center. One scenario proposed by Goicoechea et al. (2013) to explain the
Sgr A* CO SLED suggests that the hot gas responsible for the high- $J_{u}$ emission is concentrated in small dense clumps that reside in a more diffuse, extended medium which gives rise to the lower $J_{u}$ emission. Another study by Kamenetzky et al. (2014) compared the CO SLEDs of Sgr B2(M), Sgr B2(N), and the Sgr B2 envelope determined from SPIRE observations (Etxaluze et al. 2013), to those of several other galaxies. They note that the CO SLED of the Sgr B2 envelope resembles those of other galaxies, while the CO SLEDs in the Sgr B2 cores (i.e., those specifically focused on hot gas) peak at higher $J_{u}$. Furthermore, Kamenetzky et al. (2014) conclude that while $\mathrm{CO}$ emission from star-forming cores must be present in their observations of other galaxies, the line flux is dominated by emission from warm, extended molecular clouds. These large reservoirs of warm, dense gas are what make many of these galaxies exceptional, as they require some heating mechanism(s) capable of operating over extensive regions (Papadopoulos et al. 2012). In contrast, the observations of Galactic sources are tightly focused on known energetic regions, which are themselves quite compact. The full PACS footprint covers an area of about $0.1 \times 0.1 \mathrm{pc}$ in the Orion starforming region $(d \approx 420 \mathrm{pc})$, and about $2.5 \times 2.5 \mathrm{pc}$ in $\mathrm{W} 49 \mathrm{~N}$ $(d=11.1 \pm 0.8 \mathrm{kpc}$; Zhang et al. 2013). With hot, dense gas filling a large portion of the beam and a lack of "contamination" from unassociated cooler gas, the Galactic CO SLEDs peak at higher $J_{u}$.

\section{Summary}

We have observed rotational transitions from the $5 \leqslant J_{u} \leqslant 16$ states of CO with HIFI and from $J_{u} \geqslant 14$ with PACS in emission toward Orion S, Orion KL, Sgr B2(M), and W49N. Fluxes are extracted from the $\mathrm{CO}$ emission lines and used to construct SLEDs. Our original intent was to empirically interpret CO SLEDs in other galaxies by reconstructing them from linear combinations of CO SLEDs in Galactic sources where the gas properties and heating mechanisms are well characterized. However, the CO SLEDs in our sample of Galactic sources all peak at higher $J_{u}$ than the CO SLEDs observed in other galaxies, such that no combination can successfully reproduce the extragalactic observations. The difference between Galactic and extragalactic CO SLEDs is primarily a beam filling effect. Our observations in the Milky Way specifically target starforming cores, preferentially sampling hot molecular gas while excluding cold quiescent gas, such that the resulting CO SLEDs peak around $14 \lesssim J_{u} \lesssim 20$. In other galaxies the PACS and SPIRE beams cover much larger physical regions than they do within the Milky Way. As a result, in addition to the small pockets of hot, dense gas that produce high- $J_{u} \mathrm{CO}$ emission, these beams also sample a large amount of cooler, more extended gas. It is this material, filling a much larger portion of the beam than the hot dense gas, which dominates the $\mathrm{CO}$ emission and causes SLEDs to peak closer to $J_{u} \sim 8$. As such, we urge that careful consideration be given to these effects when comparing Herschel observations sampling vastly different physical size scales.

The authors thank the anonymous referee for suggestions to improve the clarity of the paper. Support for this work was provided by NASA through an award issued by JPL/Caltech. J. R.G. and J.C. thank the ERC for support under grant ERC-2013Syg-610256-NANOCOSMOS, and the Spanish MINECO for support under grant AYA2012-32032. HIFI has been designed 
and built by a consortium of institutes and university departments from across Europe, Canada, and the United States under the leadership of SRON Netherlands Institute for Space Research, Groningen, The Netherlands and with major contributions from Germany, France and the US. Consortium members are Canada: CSA, U. Waterloo; France: CESR, LAB, LERMA, IRAM; Germany: KOSMA, MPIfR, MPS; Ireland, NUI Maynooth; Italy: ASI, IFSI-INAF, Osservatorio Astrofisico di Arcetri-INAF; Netherlands: SRON, TUD; Poland: CAMK, CBK; Spain: Observatorio Astronmico Nacional (IGN), Centro de Astrobiologa (CSIC-INTA). Sweden: Chalmers University of TechnologyMC2, RSS \& GARD; Onsala Space Observatory; Swedish National Space Board, Stockholm University-Stockholm Observatory; Switzerland: ETH Zurich, FHNW; USA: Caltech, JPL, NHSC.

PACS has been developed by a consortium of institutes led by MPE (Germany) and including UVIE (Austria); KU Leuven, CSL, IMEC (Belgium); CEA, LAM (France); MPIA (Germany); INAF-IFSI/OAA/OAP/OAT, LENS, SISSA (Italy); IAC (Spain). This development has been supported by the funding agencies BMVIT (Austria), ESA-PRODEX (Belgium), CEA/CNES (France), DLR (Germany), ASI/INAF (Italy), and CICYT/MCYT (Spain).

\section{References}

Alves, J., \& Homeier, N. 2003, ApJL, 589, L45

Bergin, E. A., Phillips, T. G., Comito, C., et al. 2010, A\&A, 521, L20

Blake, G. A., Sutton, E. C., Masson, C. R., \& Phillips, T. G. 1987, ApJ, 315,621

Bland-Hawthorn, J., Gallimore, J. F., Tacconi, L. J., et al. 1997, Ap\&SS, 248, 9 Braatz, J. A., Wilson, A. S., \& Henkel, C. 1997, ApJS, 110, 321

Crockett, N. R., Bergin, E. A., Neill, J. L., et al. 2014, ApJ, 787, 112

Dalcanton, J. J., Williams, B. F., Seth, A. C., et al. 2009, ApJS, 183, 67

de Graauw, T., Helmich, F. P., Phillips, T. G., et al. 2010, A\&A, 518, L6

Draine, B. T. 1978, ApJS, 36, 595

Etxaluze, M., Goicoechea, J. R., Cernicharo, J., et al. 2013, A\&A, 556, A137

Galván-Madrid, R., Liu, H. B., Zhang, Z.-Y., et al. 2013, ApJ, 779, 121

Gerin, M., Ruaud, M., Goicoechea, J. R., et al. 2015, A\&A, 573, A30

Goicoechea, J. R., Chavarría, L., Cernicharo, J., et al. 2015, ApJ, 799, 102

Goicoechea, J. R., Etxaluze, M., Cernicharo, J., et al. 2013, ApJL, 769, L13

Griffin, M. J., Abergel, A., Abreu, A., et al. 2010, A\&A, 518, L3

Hailey-Dunsheath, S., Sturm, E., Fischer, J., et al. 2012, ApJ, 755, 57

Henney, W. J., O’Dell, C. R., Zapata, L. A., et al. 2007, AJ, 133, 2192

Janssen, A. W., Bruderer, S., Sturm, E., et al. 2015, ApJ, 811, 74

Kamenetzky, J., Glenn, J., Rangwala, N., et al. 2012, ApJ, 753, 70
Kamenetzky, J., Rangwala, N., Glenn, J., Maloney, P. R., \& Conley, A. 2014, ApJ, 795, 174

Kristensen, L. E., van Dishoeck, E. F., Bergin, E. A., et al. 2012, A\&A, 542, A8

Mashian, N., Sturm, E., Sternberg, A., et al. 2015, ApJ, 802, 81

Meijerink, R., Kristensen, L. E., Weiß, A., et al. 2013, ApJL, 762, L16

Menten, K. M., Reid, M. J., Forbrich, J., \& Brunthaler, A. 2007, A\&A, 474,515

Murakami, H., Koyama, K., Sakano, M., Tsujimoto, M., \& Maeda, Y. 2000, ApJ, 534, 283

Nagy, Z., Choi, Y., Ossenkopf-Okada, V., et al. 2017, A\&A, in press (arXiv:1611.07470)

Nagy, Z., van der Tak, F. F. S., Fuller, G. A., Spaans, M., \& Plume, R. 2012, A\&A, 542, A6

Nagy, Z., Van der Tak, F. F. S., Ossenkopf, V., et al. 2013, A\&A, 550, A96

Neill, J. L., Bergin, E. A., Lis, D. C., et al. 2014, ApJ, 789, 8

Nissen, H. D., Cunningham, N. J., Gustafsson, M., et al. 2012, A\&A, 540, A119

O’Dell, C. R., Muench, A., Smith, N., \& Zapata, L. 2008, in Handbook of Star Forming Regions, Vol. 1, ed. B. Reipurth (San Francisco, CA: ASP), 544

Ott, S. 2010, in ASP Conf. Ser. 434, Astronomical Data Analysis Software and Systems XIX, ed. Y. Mizumoto, K.-I. Morita, \& M. Ohishi (San Francisco, CA: ASP), 139

Papadopoulos, P. P., van der Werf, P. P., Xilouris, E. M., et al. 2012, MNRAS, 426, 2601

Peng, T.-C., Wyrowski, F., Zapata, L. A., Güsten, R., \& Menten, K. M. 2012, A\&A, 538, A12

Pilbratt, G. L., Riedinger, J. R., Passvogel, T., et al. 2010, A\&A, 518, L1

Poglitsch, A., Waelkens, C., Geis, N., et al. 2010, A\&A, 518, L2

Rivilla, V. M., Martín-Pintado, J., Sanz-Forcada, J., Jiménez-Serra, I., \& Rodríguez-Franco, A. 2013, MNRAS, 434, 2313

Roberts, H., van der Tak, F. F. S., Fuller, G. A., Plume, R., \& Bayet, E. 2011, A\&A, 525, A107

Rosenberg, M. J. F., van der Werf, P. P., Aalto, S., et al. 2015, ApJ, 801, 72

San José-García, I., Mottram, J. C., van Dishoeck, E. F., et al. 2016, A\&A, 585, A103

Schmid-Burgk, J., Guesten, R., Mauersberger, R., Schulz, A., \& Wilson, T. L. 1990, ApJL, 362, L25

Sievers, A. W., Mezger, P. G., Bordeon, M. A., et al. 1991, A\&A, 251, 231

Spinoglio, L., Pereira-Santaella, M., Busquet, G., et al. 2012, ApJ, 758, 108

Tahani, K. 2013, Master's thesis, Univ. of Calgary

Tahani, K., Plume, R., Bergin, E. A., et al. 2016, ApJ, 832, 12

van der Wiel, M. H. D., van der Tak, F. F. S., Ossenkopf, V., et al. 2009, A\&A, 498, 161

Wu, S.-W., Bik, A., Bestenlehner, J. M., et al. 2016, A\&A, 589, A16

Zapata, L. A., Ho, P. T. P., Rodríguez, L. F., et al. 2006, ApJ, 653, 398

Zapata, L. A., Rodríguez, L. F., Ho, P. T. P., et al. 2005, ApJL, 630, L85

Zapata, L. A., Schmid-Burgk, J., Ho, P. T. P., Rodríguez, L. F., \& Menten, K. M. 2009, ApJL, 704, L45

Zapata, L. A., Schmid-Burgk, J., \& Menten, K. M. 2011, A\&A, 529, A24

Zhang, B., Reid, M. J., Menten, K. M., et al. 2013, ApJ, 775, 79

Ziurys, L. M., Wilson, T. L., \& Mauersberger, R. 1990, ApJL, 356, L25 